Received: 4 May 2018

Accepted: 12 December 2018

Published online: 31 January 2019

\section{A pilot, open labelled, randomised controlled trial of hypertonic saline nasal irrigation and gargling for the common cold}

\author{
Sandeep Ramalingam (10 ${ }^{1}$, Catriona Graham ${ }^{2}$, Jenny Dove ${ }^{1}$, Lynn Morrice ${ }^{3}$ \& Aziz Sheikh ${ }^{3}$
}

There are no antivirals to treat viral upper respiratory tract infection (URTI). Since numerous viruses cause URTI, antiviral therapy is impractical. As we have evidence of chloride-ion dependent innate antiviral response in epithelial cells, we conducted a pilot, non-blinded, randomised controlled trial of hypertonic saline nasal irrigation and gargling (HSNIG) vs standard care on healthy adults within 48 hours of URTI onset to assess recruitment (primary outcome). Acceptability, symptom duration and viral shedding were secondary outcomes. Participants maintained a symptom diary until well for two days or a maximum of 14 days and collected 5 sequential mid-turbinate swabs to measure viral shedding. The intervention arm prepared hypertonic saline and performed HSNIG. We recruited 68 participants (2.6 participants/week; November 2014-March 2015). A participant declined after randomisation. Another was on antibiotics and hence removed (Intervention:32, Control:34). Follow up data was available from 61 (Intervention:30, Control:31). 87\% found HSNIG acceptable, 93\% thought HSNIG made a difference to their symptoms. In the intervention arm, duration of illness was lower by 1.9 days $(p=0.01)$, over-the-counter medications (OTCM) use by $36 \%(p=0.004)$, transmission within household contacts by $35 \%(p=0.006)$ and viral shedding by $\geq 0.5 \log _{10} /$ day $(p=0.04)$. We hence need a larger trial to confirm our findings.

The common cold is a viral upper respiratory tract infection (URTI). Adults and children get 2-3 and 6-7 attacks respectively of URTI annually ${ }^{1,2}$. In 2016 the UK lost 34.0 million work days (33.1\% of total) due to minor illnesses such as URTI ${ }^{3}$. An episode of URTI cost $€ 266.41, € 273.36$ in Cardiff and Southampton respectively ${ }^{4}$. In the US, $72 \%$ of respondents had URTI in the past year costing $\$ 40$ billion annually ${ }^{2}$. Outbreaks of respiratory tract infections are common in hospitals and care homes with significant morbidity and mortality ${ }^{5}$. URTI can lead to lower respiratory tract infections (LRTI) such as pneumonia, or cause exacerbations in individuals with asthma, chronic obstructive pulmonary disease (COPD), and cystic fibrosis ${ }^{6}$. Since URTI precedes LRTI, early intervention could prevent these complications.

At present, there are no antiviral agents to treat the common cold. Though rhinovirus is called the "common cold virus", a large number of viruses cause URTI ${ }^{7}$. Hence, specific antiviral treatment is impractical, and we need an intervention effective against multiple viruses. Jalaneti (cleaning the nasal passages with salt water), an ancient practice from India is recommended in Yogic texts for the common cold ${ }^{8}$. A significant reduction in sore throats and colds was reported when Australian wood-workers performed Jalaneti for a year ${ }^{9}$. Three randomised controlled trials (RCT) report the efficacy of salt water for acute URTI. Adam et al. reported the lack of improvement in symptoms or duration of illness in adults with a common cold or bacterial rhinosinusitis [hypertonic saline (HS)/normal saline (NS) sprays thrice/day vs. standard care]. However, individuals with a common cold who received HS sprays said they would use it again $(\mathrm{p}=0.007)^{10}$. Sea-water sprays (six-times/day) significantly reduced sore throat, nasal secretions, decongestant/mucolytic use in children with URTI ${ }^{11}$. A Cochrane review

${ }^{1}$ Department of Laboratory Medicine, Royal Infirmary of Edinburgh, 51 Little France Crescent, Edinburgh, EH16 4SA, UK. ${ }^{2}$ Wellcome Trust Clinical Research Facility, University of Edinburgh, Western General Hospital, Crewe Road South, Edinburgh, EH4 2XU, UK. ${ }^{3}$ Centre of Medical Informatics, Usher Institute of Population Health Sciences and Informatics, The University of Edinburgh, Medical School Doorway 3, Teviot Place, Edinburgh, EH8 9AG, UK. Correspondence and requests for materials should be addressed to S.R. (email: sandeep.ramalingam@nhslothian. scot.nhs.uk) 


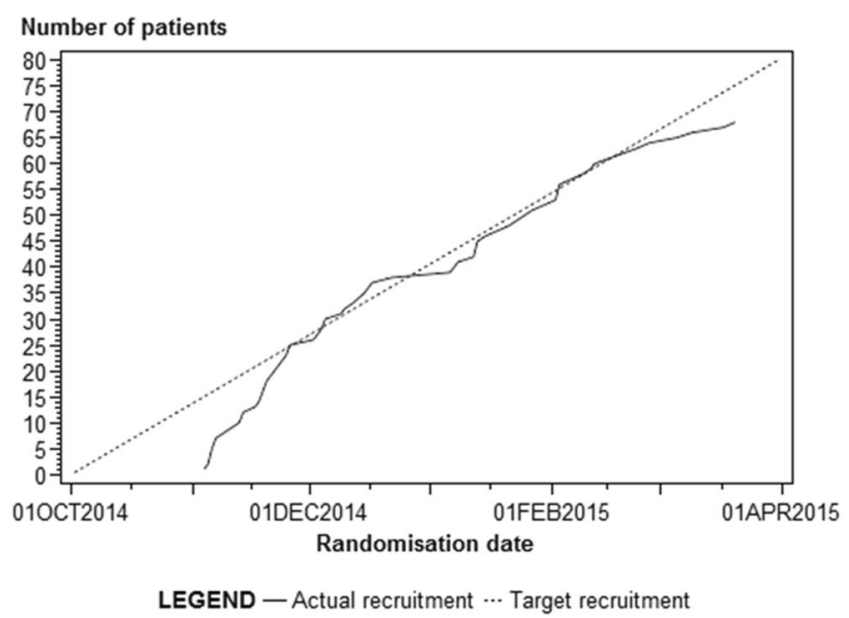

Figure 1. Expected and actual recruitment: Recruitment of participants from the 1st October 2014 till close of recruitment on 31st March 2015. If we take the full 26-week period, this would result in an average of 2.6 participants per week over the study period. However, if we were to take the first and last recruitment dates this give a period of 20 weeks which results in an average of 3.4 participants per week.

concludes that the evidence is very limited ${ }^{12}$. In a recent report, both NS and sea-water drops (thrice/day) reduced the severity of URTI symptoms in young children ${ }^{13}$.

Inhibition of viral replication in the presence of chloride/halide salts was reported in the 1960 's $^{14}$. We have recently reported laboratory evidence that non-myeloid cells (e.g. epithelial, fibroblast and hepatic cells) have an innate immune mechanism, which is augmented in the presence of salt $(\mathrm{NaCl})^{15}$. In cell culture models, DNA, RNA, enveloped and non-enveloped viruses are all inhibited in the presence of $\mathrm{NaCl}^{15}$. The antiviral effect is dependent on the availability of chloride ions (and not sodium ions) ${ }^{15}$. In the presence viral infection and the availability of $\mathrm{NaCl}$, cells utilise the chloride ions to produce hypochlorous acid $(\mathrm{HOCl})^{15}$. Since $\mathrm{HOCl}$ is the active ingredient in bleach, which is known to have an antiviral effect, the mechanism could be augmented by supplying chloride ions through $\mathrm{NaCl}$ to treat infections. Here, we report the results of the Edinburgh and Lothians Viral Intervention Study (ELVIS), a pilot RCT of hypertonic saline nasal irrigation and gargling (HSNIG) versus standard care in adults with URTI to determine if we can recruit and retain participants in Edinburgh and to get initial information on acceptability, duration of symptoms, and viral shedding.

\section{Results}

We recruited 68 participants over 26 weeks between October 2014 and March 2015 (2.6 participants/week: Fig. 1). We excluded two $(\mathrm{n}=66)$ [one declined after randomisation, and the other was on antibiotics] and randomised 32 to the intervention arm and 34 to the control arm (Fig. 2). The majority (76\%) were women. Most (76\%) preferred paper forms over online feedback. Most (Intervention: 94\%, Control: 91\%) returned the daily forms, end-of-study form (Intervention: 88\%, Control: 85\%) and swabs (Intervention: $88 \%$, Control: $91 \%$ ). Of the sixty-six participants, five did not return daily forms. Of the sixty-one, four did not return end-of-study forms. Of the fifty-seven, three did not return samples. All forms and samples were hence available in fifty-four individuals.

$80 \%$ in the intervention arm used $3 \% \mathrm{HS}$, while $9 \%$ each used $2.5 \%, 2 \% \mathrm{HS}$. All but one used Cornish sea salt. One participant left without the sea salt and used another brand available at home. An individual in the control arm reported performing nasal irrigation but provided no further information.

The baseline characteristics, symptom severity and degree of interference with daily life were similar in both arms (Table 1). We identified an aetiology in the baseline sample of $73 \%$ (48/66) of participants (Table S1). Amongst these, $56 \%$ were rhinovirus and $31 \%$ were coronaviruses (COV), with the rest due to enterovirus, influenza A virus, parainfluenza virus type 3 (PIV-3), respiratory syncytial virus (RSV) and human metapneumovirus (HMPV). We detected dual infections of rhinovirus with an enterovirus/COV HKU1 in two.

Most participants completed the "WURSS-21-Scot" daily until they were well (i.e. a score of 0) on two days (please see Fig. S1 for daily forms). Intervention and control arms completed the symptom diary for a mean (SD) of 6.8 (2.2) and 8.7 (3.3) days respectively. The intervention arm hence had a reduction in duration of illness by 1.9 days $(95 \% \mathrm{CI}=0.4$ to 3.3$)(\mathrm{p}=0.01)$ (Fig. 3 ). The duration of illness was significantly lower $(\mathrm{p}=0.01)$ even if the first day participants felt well was the end-point [Mean (SD) Intervention: 6.0 (2.4); Control: 8.0 (3.4) days]. Participants performed HSNIG for a median of 5 days (IQR: 3 to 6 ) and at a median of thrice a day (IQR: 2 to 3 ) (Fig. 3).

There was a significant reduction in the duration of runny nose ( 1.8 days, $95 \%$ CI:0.4 to $3.2, \mathrm{p}=0.01)$, blocked nose (2.7 days, $95 \%$ CI:1.2 to 4.1, $\mathrm{p}<0.001$ ), sneezing ( 1.5 days, $95 \%$ CI:0.3 to $2.9, \mathrm{p}=0.02$ ), cough (2.4 days, 95\% CI:0.9 to $4.0, \mathrm{p}=0.003$ ), hoarseness of voice (1.7 days, $95 \%$ CI:0.2 to 3.1, $\mathrm{p}=0.02$ ) (Fig. 3; Supplementary Table S2).

We could calculate the average WURSS-21 score and EQ-VAS scores from the diaries (please also see supplementary results). The median (IQR) average WURSS 21 score in the intervention group was 13.2 (7.6 to 16.4) $[n=30]$ and 16.9 (9.9 to 24.7$)[n=31]$ in the control arm $(p=0.09)$. The mean (SD) average quality of life 


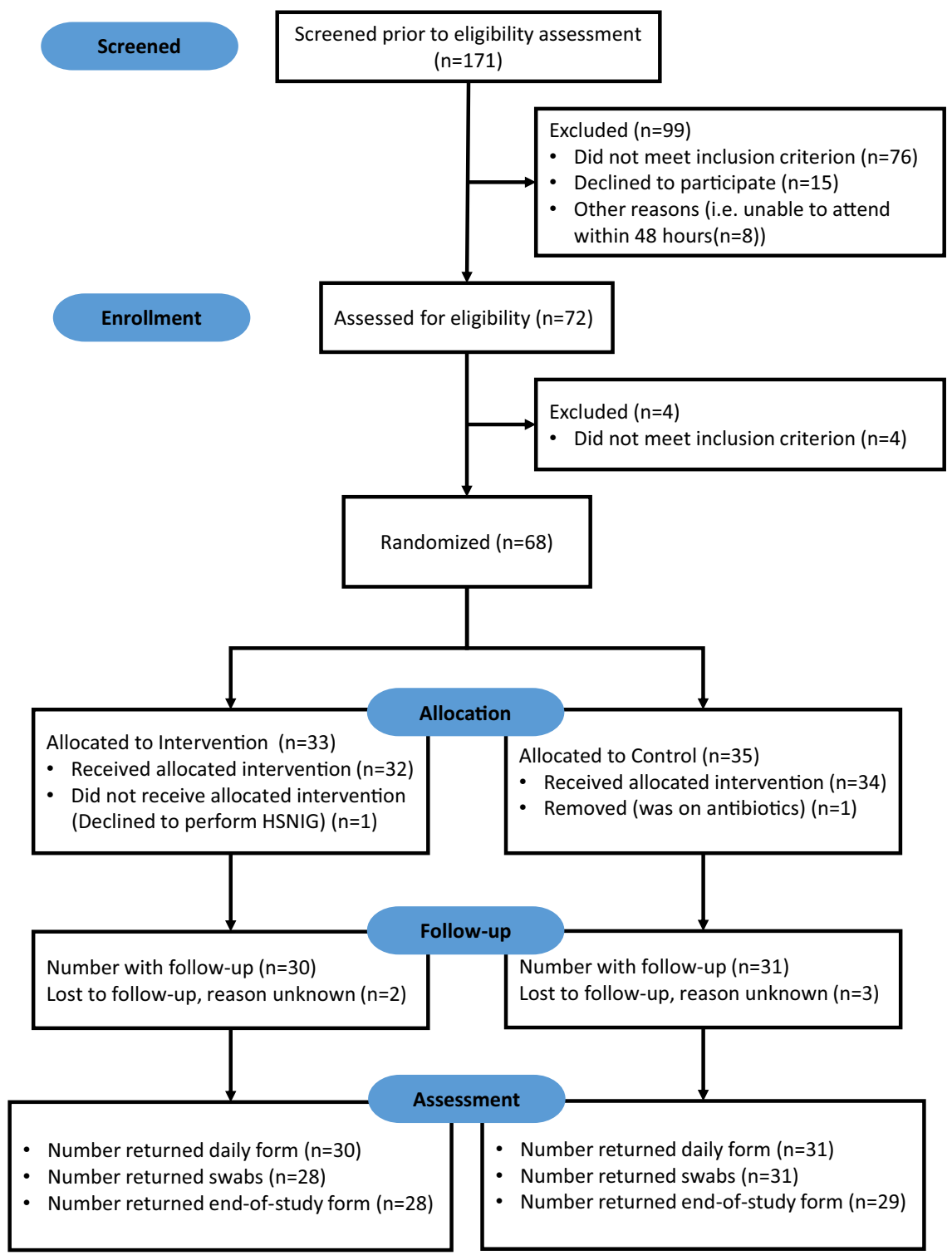

Figure 2. Consort Flow diagram: Based on the "CONSORT extension for Pilot and Feasibility Trials Flow Diagram" (http://www.consort-statement.org/downloads/extensions - Accessed 04/12/2018).

measure (EQ-VAS) over the study duration was higher at $74.3(12.1)[\mathrm{n}=30]$ for the intervention and $70.8(15.5)$ $[n=31]$ in the control arm. The difference in means of $3.4,95 \%$ CI for difference ( -3.7 to 10.6$)$ was not significant $(\mathrm{p}=0.338)$ (Supplementary Table S3).

Excluding those with no virus detected in the baseline sample [Intervention: 5 (16\%); Control: 12 (35\%)], samples on days 1-4 to estimate viral shedding was available in 25 and 20 individuals in intervention and control arms respectively (Fig. 4). In four individuals [a COV and a rhinovirus each per arm], no virus was detectable on days 1-4. Details of symptom severity, viral shedding and HSNIG are in Fig. 4.

The median (IQR) reduction in viral shedding between baseline and end-point sample in the intervention arm was $-2.23 \log _{10}(-3.04$ to -0.32$)[\mathrm{n}=26]$ and $-1.51 \log _{10}(-3.30$ to -0.55$)[\mathrm{n}=21]$ in the control arm $(\mathrm{p}=0.9)$. (see supplementary results). We then estimated the proportion of individuals with viral shedding by $\geq 0.5 \log _{10}$ /day between arms (see supplementary results). Four individuals in the intervention arm had paramyxovirus infection but none in the control arm. As paramyxoviruses have a longer life cycle ${ }^{16,17}$, incubation period ${ }^{18}$, duration of viral shedding ${ }^{19}$ and illness ${ }^{19,20}$ compared to rhinovirus ${ }^{21-24}$, the data was analysed after removing these individuals. A higher proportion in the intervention arm had a fall $\geq 0.5 \log _{10} /$ day compared to controls [Intervention 73\% [ $n=16 / 22]$, Control 43\% [ $n=9 / 21]$, difference $-30 \%, 95 \%$ CI for difference in proportion $(-58$ to -2$) \mathrm{p}=0.04]$.

End-of-study forms were available from fifty-seven individuals (Table 2). Over-the-counter medications use (OTCM) use was $36 \%$ lower in the intervention arm (95\% CI 14 to 59) $(\mathrm{p}=0.004)$. Amongst participants who were not living alone, $35 \%$ fewer individuals in the intervention arm (95\% CI 10 to 60) had household contacts developing URTI after them $(\mathrm{p}=0.006)$. 


\begin{tabular}{|l|c|c|}
\hline & Intervention $(\mathbf{n}=\mathbf{3 2}) \mathbf{n}(\%)$ & Control $(\mathbf{n}=\mathbf{3 4}) \mathbf{n}(\%)$ \\
\hline Age mean(SD) & $34.6(9.3)$ & $39.4(10.9)$ \\
\hline WURSS-21 Score mean (SD) & $41.6(18.2)$ & $43.9(21.8)$ \\
\hline EQ-VAS Score mean(SD) & $65.9(13.6)$ & $63.7(17.4)$ \\
\hline Sex (Female) & $24(75)$ & $25(74)$ \\
\hline Tobacco smoker - current & $1(3)$ & $3(9)$ \\
\hline Tobacco smoker - ex & $5(16)$ & $11(32)$ \\
\hline e-Cigarette smoker - current & $0(1)$ & $1(3)$ \\
\hline e-Cigarette smoker - ex & $2(6)$ & $0(0)$ \\
\hline Adults at home $=1$ & $6(19)$ & $5(15)$ \\
\hline \multicolumn{1}{|c|}{$>1$} & $26(81)$ & $29(85)$ \\
\hline Children at home $=0$ & $19(59)$ & $19(56)$ \\
\hline \multicolumn{1}{|c|}{1} & $7(22)$ & $5(15)$ \\
\hline$>1$ & $6(19)$ & $10(29)$ \\
\hline No one unwell before them at home & $19(59)$ & $21(62)$ \\
\hline Employment status: Full-time & $20(63)$ & $21(62)$ \\
\hline \multicolumn{1}{|c|}{ Part-time } & $7(22)$ & $4(15)$ \\
\hline Education: Full time & $4(13)$ & $4(12)$ \\
\hline Other & $1(3)$ &
\end{tabular}

Table 1. Baseline characteristics. WURSS: Wisconsin upper respiratory symptom survey; EQ-VAS: EuroQolVisual Analog Scale.

We received feedback on HSNIG from 28/32 participants (Table S4). 93\% said that HSNIG made a difference to their symptoms. They found HS easy to prepare (100\%). Most preferred to prepare solution in bulk in a flask $(86 \%)$ and preferred using a small bowl to perform HSNIG (75\%). Most considered the procedure either comfortable or moderately comfortable (89\%) and the majority (86\%) were happy to perform the procedure outside home. The most commonly reported effects were that it helped clear the nose/reduced stuffiness $(n=16)$, made it easier to breathe $(n=7)$, speeded up resolution of symptoms $(n=5)$, reduced the severity of the illness $(n=3)$ and improved sleep $(n=2) .60 \%$ of participants were likely to use HSNIG in the future and $25 \%$ of participants were undecided if they would use HSNIG in the future. The figure increased to $86 \%$ if the procedure could be made more convenient. Most (71\%) however said they were unlikely to use HSNIG as a prophylactic measure.

\section{Discussion}

Our pilot RCT confirms that it's possible to recruit and retain participants for a full trial of HSNIG with 3\% HS. HSNIG reduced the duration of illness (22\%), OTCM use (36\%) and illness in household members (35\%). When individuals infected with similar viruses (rhinovirus, coronavirus, enterovirus and influenza virus) were compared, $30 \%$ more individuals had reduction in viral shedding by $\geq 0.5 \log _{10}$ per day in the intervention arm. This could explain both the reduction in the duration of illness and transmission to household contacts in the intervention arm. However, though the difference between the baseline and end-point samples was larger in the intervention arm than the control arm, the difference was not significant (although this study was not powered to detect differences in these measures). In participants who stopped HSNIG before day four, 54\% (7/13) had an increase in viral shedding. There was also an increase/stabilisation of symptoms in $50 \%(8 / 16)$ before symptoms resolved. In fact, four individuals felt the need to restart HSNIG for one or more days (Fig. 4). These finding along with the lower rate of symptomatic household contacts in the intervention arm suggest that HSNIG helps reduce viral replication. Since viruses are shed during breathing and speaking ${ }^{7}$, measure that helps reduce viral shedding would help reduce transmission.

Recruitment was relatively easy though it involved regular email reminders. Advertising through social media could potentially help in future studies. A major concern was whether the population in Edinburgh would be happy to perform HSNIG. Surprisingly, only one individual declined to participate having met the trial nurse. Patient reported compliance with HSNIG was excellent. Participants performed HSNIG more times in the earlier part of the URTI and fewer times as symptoms improved, a trend which was in keeping with the severity of illness (Fig. 3). A surprisingly high proportion (86\%) reported performing HSNIG outside their homes. 93\% found HSNIG useful and $61 \%$ said they would perform HSNIG again if they had a cold, with a higher uptake if the procedure was more convenient. Alternatives such as nasal sprays are options, though they would not have the physical rinsing component of nasal irrigation. Hence a study to compare the two methodologies would be useful.

WURSS 21 score was not significantly different between the two arms, probably a reflection in the sample size. Neither was the EQ VAS score different between arms. EQ VAS score is an indicator of how a person feels on a given day and is not specific to URTI. It is hence probably not suitable for studies on URTI.

Our study has limitations. As a pilot with a primary outcome of establishing if a trial using HSNIG is viable, the study is not powered for efficacy end-points. We hence need a larger trial to confirm our findings. The lack of a placebo group is another limitation. Since our hypothesis was that the chloride ion has an antiviral effect, we were unable to use NS as a control as it could cause a reduction in symptoms. This is supported by results from earlier studies. For e.g. in a cross over trial (10 weeks twice daily nasal spray and 10 weeks without sprays with a 

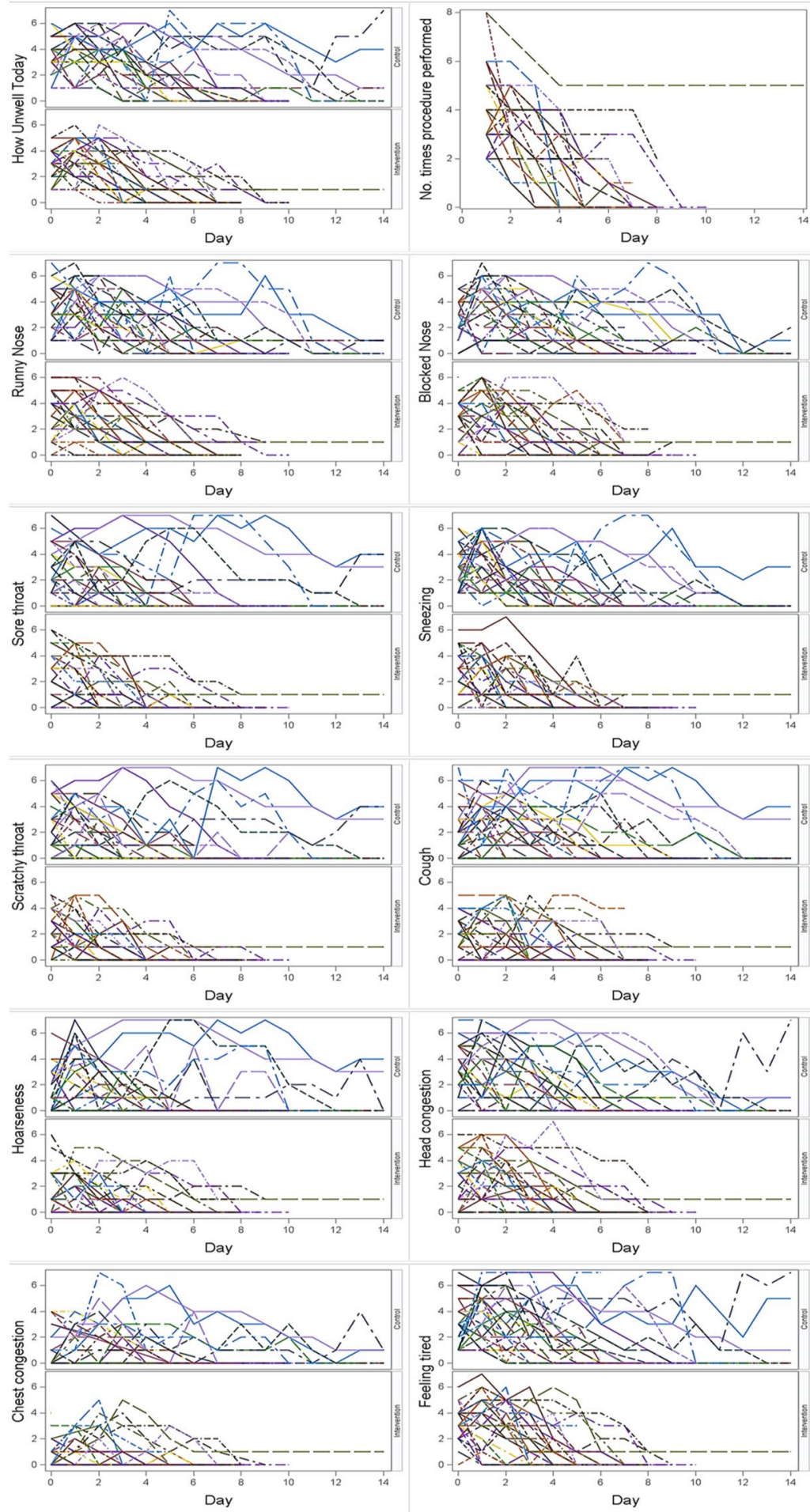

Figure 3. Response from participants over the study period: Each line represents response of a participant over 14 days. Data is shown by treatment group. The global severity question was "How unwell do you feel today". The responses were graded from 0 (Not unwell), 1 (very mildly), 3 (mildly), 5 (moderately) and 7 (severely unwell). Likewise, each symptom was graded 0 (no symptom) to 7 (severe).

two-week washout period), twice a day saline sprays significantly reduced nasal symptoms in military recruits compared to those with no intervention $(\mathrm{p}=0.027)$. The number of episodes of URTI was lower in the period when saline sprays were used compared to the period when sprays were not used. However, the difference was just short of significance $(\mathrm{p}=0.05)^{25}$. Our rationale also seems vindicated by a recent report suggesting that both sea water drops and saline drops were equally effective in treating children $<2$ years of age with URTI compared to symptomatic controls ${ }^{13}$. Sodium bicarbonate, though commonly used, is uncomfortable in the author's personal 


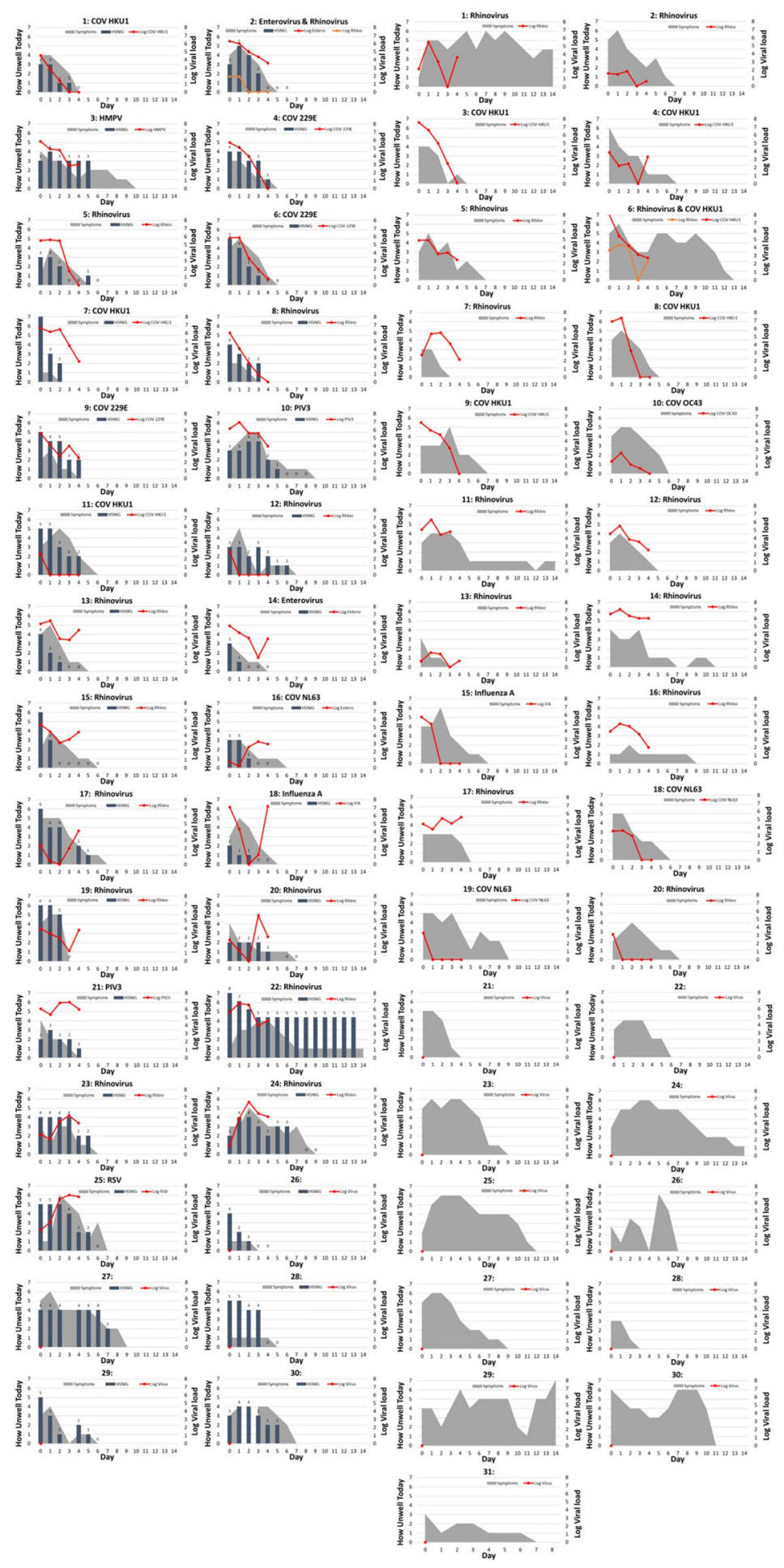

Figure 4. Severity of symptoms, viral shedding and relationship to HSNIG and participant number in each arm: For each participant, the daily scores for the global question 'how unwell do you feel today' over 14 days are represented by the grey background. For the intervention arm (shown on the left), the blue columns represent the number of times HSNIG was done that day. For both arms, change in viral shedding (as log values; Red line) is shown for the corresponding days.

experience. Recent evidence suggests that sodium ion also has an antimicrobial effect ${ }^{26,27}$. Hence, until a safe and comfortable placebo that contain neither chloride, halide or sodium ion is identified, placebo-controlled trials cannot be done. 


\begin{tabular}{|c|c|c|c|c|c|}
\hline & \multicolumn{2}{|c|}{ Intervention } & \multicolumn{2}{|c|}{ Control } & \multirow[b]{2}{*}{$\mathbf{p}$} \\
\hline & $\mathrm{n}=\mathbf{2 8}$ & $\%$ & $\mathrm{n}=\mathbf{2 9}$ & $\%$ & \\
\hline Took time off work & 3 & 11 & 7 & 24 & $0.297 *$ \\
\hline Days off work: 1 & 2 & 7 & 2 & 7 & \\
\hline 2 & 1 & 4 & 1 & 3 & \\
\hline 3 & - & 0 & 4 & 14 & \\
\hline Took over-the-counter medication & 14 & 50 & 25 & 86 & $0.004^{*}$ \\
\hline Attended GP & - & 0 & $2 * *$ & 7 & \\
\hline Attended hospital & $1 * *$ & 4 & - & 0 & \\
\hline $\begin{array}{l}\text { Household contact developed } \\
\text { symptoms after participant }\end{array}$ & $8 / 26$ & 31 & 19 & 66 & 0.006 \\
\hline Performed procedure & 28 & 100 & 1 & 3 & \\
\hline Collecting swabs: Easy & 19 & 68 & 18 & 62 & \\
\hline Moderate & 7 & 25 & 3 & 10 & \\
\hline Difficult & 2 & 7 & 8 & 28 & \\
\hline Returning swabs: Easy & 26 & 93 & 28 & 97 & \\
\hline Diary completion: Easy & 25 & 89 & 28 & 97 & \\
\hline Form completion: Easy & 25 & 89 & 27 & 93 & \\
\hline Pre-study information - Useful & 27 & 96 & 28 & 97 & \\
\hline Instruction pack - Helpful & 28 & 100 & 27 & 93 & \\
\hline HSNIG video: Helpful & 27 & 96 & - & - & \\
\hline
\end{tabular}

Table 2. Feedback from participants at the end of study. *Using fishers exact test due to small counts or expected counts, **Reason for attending GP/hospital was not available, GP: General practitioner, HSNIG Hypertonic saline nasal irrigation and gargling.

Another aspect that needs to be considered is the potential benefits of the simple process of flushing in the intervention arm. Even in the presence of a placebo arm, this cannot be answered. Further studies with different methodologies for supplying $\mathrm{NaCl}$ (e.g. hypertonic saline sprays, or aerosolised $\mathrm{NaCl}$ ) may help answer this question.

In the absence of a placebo, we focused on viral shedding as an objective measure of antiviral activity due to HSNIG. There were more individuals without a detectable virus in the baseline sample in the control arm $(12 / 34: 35 \%)$ compared to the intervention arm (5/32:16\%). This difference did not however reach statistical significance $(\mathrm{p}=0.059)$ (Table S1). Though allergic rhinitis (history of allergy with current eye/nose itching or sneezing) was an exclusion criterion, it is possible that some of these individuals could have allergic rhinitis. Or, the infective aetiology might not have been detected in the nose swab. For e.g. sore throat was often recorded by those who did not have a detectable virus. As we collected a nose swab, it is possible that the aetiology could be picked up by including a throat swab along with a nose swab.

Viral shedding is difficult to quantify due to the variability in sampling and as most routine respiratory PCR's are qualitative assays. We used self-collected mid-turbinate swabs (Copan, Italy) both for participants convenience (no gag reflex) and as the swabs are designed with a stop which increases safety and should help reduce variation in sampling ${ }^{28}$. Since nasal irrigation could physically wash off the virus, we collected swabs first thing in the morning before HSNIG. We used eNAT, a transport medium that inactivates viruses and in which samples are stable at room temperature for at least two weeks. In our hands, samples in eNAT were stable for at least a week at room temperature and could be posted back to the laboratory for testing ${ }^{29}$. Where we identified a virus in the baseline sample, we tested all five samples in the same run to minimise inter-assay variability. To compare viral shedding, we converted CT values to $\log _{10}$ values. The baseline samples were hence tested on two occasions. The inter-assay variation between the two results was very low [mean (SD): $0.21 \log _{10}(1.17)$ ]. The cut-off of $\geq 0.5 \log _{10}$ per day used to determine reduction in viral shedding is more than double that of the inter-assay variation seen and hence is unlikely to be an artefact of the testing process. All these measures together have helped produce viral shedding data that could be compared between arms. In four individuals with a positive baseline sample, follow-up samples were negative. In two individuals a sample with a low CT (i.e. high viral copy) were followed by samples with undetectable virus. Since our consent did not include human DNA testing, we could not test for housekeeping genes and cannot be certain if these samples were properly collected. This need to be addressed in future studies.

We detected viruses in $73 \%$ of individuals, much higher than $40-55 \%$ reported by others ${ }^{30-32}$. This could be due to sampling within 48 hours of onset of illness. Though rhinoviruses and coronaviruses were the commonest, our study confirms that numerous viruses cause URTI. The viral load of the initial sample varied between individuals and sequential sampling is important to detect change in viral shedding. A larger study would help determine the relative efficacy of HSNIG against different viruses.

At baseline, those infected with a virus other than rhinovirus had more individuals with runny nose and blocked nose (data not shown). Sore throat was often recorded in those without a viral aetiology. Hence a baseline throat swab for both bacteria and viruses may help determine the aetiology in these individuals. 
The results of ELVIS are significantly different to that from Adam et al. which had many methodological issues. They compared 2\% HS spray, NS spray (two squirts, thrice a day) and a control group in individuals with the common cold or rhinosinusitis ${ }^{10}$. Though sample size was similar to ELVIS (35-43/arm), individuals were recruited up to 3 weeks after illness onset. Very few had the common cold (12-17/arm), most had bacterial rhinosinusitis and $98 \%$ of them were treated with antibiotics. Despite these shortcomings, individuals with the common cold who received HS sprays said they would use it again $(\mathrm{p}=0.007)$. To avoid these shortcomings, we selected only those with a common cold within 48 hours of onset, and who were not on antibiotic therapy.

Strengths of the study are the use of WURSS-21, a validated symptom score diary ${ }^{33}$, for up to two weeks, and sequential sampling over 5 days in otherwise healthy adults. With positive results in a controlled population, we can also look at more challenging population groups in subsequent studies. An alternative strategy would be to use HSNIG as a prophylactic tool. Wood workers who performed nasal irrigation twice a day for a year had fewer episodes of sore throat and colds ${ }^{9}$. Sea water sprays thrice a day, for 12 weeks in children significantly reduced reported illness, school absence and use of medication ${ }^{11}$. Though feedback regarding the use of HSNIG as a prophylactic tool was negative in our population, it may not be reflective of a population at high-risk for complications such as those with asthma/COPD.

Compliance was excellent in our study. We had online videos for preparation of hypertonic saline, performing HSNIG and collection of swabs both for providing instruction to participants and as a handy reminder if needed later on. Participants were all encouraged to prepare the solution and perform HSNIG in the presence of the trial nurse which we believe helped with compliance. Participants were also trained to collect the nose swab by the trial nurse. Hence a pragmatic approach (i.e. patient reported compliance) can be taken to reduce the burden to the participant and the cost of the study. However, in patient groups where compliance might be an issue, compliance could be improved by using video monitoring of the procedures (HSNIG and collecting nasal swabs) via smart phones with support from the clinical team similar to the approach used for tuberculosis treatment ${ }^{34,35}$. The amount of salt used could also be measured at the end of the study. Tests for human DNA could be done to determine if swabs were actually collected before being introduced into the transport medium. Online data entry could be encouraged which would help reduce missing/incorrect data. Reminder messages (by text or email) could be sent to prompt regular data entry and return of samples. However, these decisions would need to be taken considering the population, the burden to the participant and the cost involved.

In this pilot, HSNIG significantly reduced the duration of URTI, OTCM use and illness within the household. A greater fall in viral shedding possibly explains the reduction in duration of symptoms and in symptomatic household contacts. This is in keeping with the lab evidence that cells utilise $\mathrm{NaCl}$ to mount an antiviral effect. A larger study powered for clinical and virological end-points is urgently needed to confirm these findings.

\section{Methods}

Study Setting and Design. We obtained ethical permission from the South-East Scotland Research Ethics Committee (13/SS/0079) and carried out the study in accordance with the Declaration of Helsinki (Clinical trials registration: NCT02438579, May 8, 2015). ELVIS was a pilot unblinded RCT of HSNIG to determine recruitment in Edinburgh as the primary outcome. Acceptability and compliance with HSNIG, quality of life, duration of symptoms and viral shedding were secondary outcomes. With a sample size of 27 per group we would be able to express the proportion of those who return the symptom score diary and samples within that group to within $\pm 19 \%$ based on a two-sided $95 \%$ confidence interval around an expected proportion of 0.5 . With the two groups combined [i.e. a sample size of 54] we would be able to express a proportion to within $\pm 13 \%$. To allow for dropouts we planned to recruit up to 80 participants to have at least 30 participants per arm for analysis. The study was advertised through schools, libraries and general practices in Edinburgh and Midlothian areas, local newspaper coverage, emails within NHS Lothian, online, social media and the study website (www.elvisstudy.com).

Identification of individuals with a cold. Individuals with URTI were identified as done by Barrett et al. ${ }^{33,36}$ Volunteers had to (1) answer "Yes" to "do you have a cold?" or "do you think you are coming down with a cold?"; (2) have at least one of first four symptoms: nasal discharge, nasal obstruction, sneezing, sore throat, headache, malaise, chilliness and cough and (3) have a Jackson Score of $\geq 2$. Onset of URTI $>48$ hours, concurrent antibiotic use, pregnancy, known chronic conditions, immunosuppression, allergic rhinitis, inability to perform HSNIG and taking part in another medical trial were exclusion criterion. Volunteers met a trial nurse at a Clinical Research Facility at the Royal Infirmary of Edinburgh (RIE) or the Western General Hospital where informed consent was obtained, and participants were then randomised. Participants were centrally allocated into intervention and control arms using a minimisation algorithm containing sex and smoking status (current/not a current smoker) with a built-in random component to ensure allocation concealment.

Feedback. Participants had to maintain a daily form (Fig. S1) until they recorded "not unwell" (i.e. score of 0) on two consecutive days or for a maximum of 14 days or until the individual needed further treatment for URTI and then filled the end-of-study form (Fig. S2). The short form of the Wisconsin Upper Respiratory Symptom Survey (WURSS-21) was used to collect daily symptom data ${ }^{33}$. For use in the Scottish context, and with the authors consent, the words "sick" and "plugged" were replaced with "unwell" and "blocked", respectively (WURSS-21-Scot) (Fig. S1). Participants were asked to answer the global severity question: "How unwell do you feel today?" which was scored from 0 (not unwell) to 7 (severely unwell). If they scored $>0$, then symptoms and functional ability were graded 0-7. They then answered the global change question "Compared to yesterday, I feel my cold is" which was scored from 0 (very much better) to 6 (very much worse). Total WURSS-21 score was calculated by adding the scores for all except the first and last question. To calculate the mean WURSS-21 score, the scores for each participant were added and divided by 14 . A mean value of EQ-VAS was calculated for each participant over the time questionnaires were returned as it was possible that participants may not score 100 even when a person's cold 
symptoms have completely resolved. Both arms documented OTCM use and if they contacted the general practitioner (GP)/nurse for further management of their URTI. Feedback about trial procedures, acceptability, health service use, costs to the patient and suggestions for improving the study and information on symptomatic household contacts were collected at the end of the study. Participants had the option to fill the daily and end-of-study forms online or on paper and return them to the laboratory.

Study Procedures. Intervention arm were taught to prepare the hypertonic saline, perform HSNIG and documented the number of times/day and side-effects. They could either prepare $100 \mathrm{ml}$ of hypertonic saline for a single use or prepare in bulk in a clean flask for use during the day. Instructions on how to make the solution and perform HSNIG are in Supplementary Methods and in www.elvisstudy.com. Control arm managed URTI as they normally did. As the hypothesis was chloride ion mediated antiviral effect, normal saline could not be used as a placebo. As sodium bicarbonate was uncomfortable in the authors personal experience, we opted for not including a placebo arm. The rationale for not including a saline placebo arm is elaborated in the discussion. Participants documented OTCM use and were asked to contact to their GP if unwell. The trial nurse helped participants in the intervention arm identify the highest concentration of HS they were comfortable with (from $3 \%, 2.5 \%, 2.0 \%$ and $1.5 \%$ ). Intervention arm were taught how to prepare HS and perform HSNIG with videos (www.elvisstudy.com) and given the opportunity to perform HSNIG under supervision. Cornish sea salt, digital-measuring spoon, bowls and flask were provided with instructions to perform HSNIG as many times as required (expected frequency up to 6 times/day for the first two days, reducing in frequency from day 3 as symptoms improved).

Mid-turbinate swab collection. The trial nurse collected a mid-turbinate swab (day 0 ) and taught participants on how to collect samples first thing in the morning on days 1-4 (before HSNIG was performed that day in the intervention arm). Flocked mid-turbinate swabs and eNAT transport medium (Copan, Italy) and Royal Mail Safebox were provided with instructions to package and return the samples to the Department of Laboratory Medicine, RIE for testing. Instructions on how to collect and return mid-turbinate swabs are in Supplementary Methods.

Virological testing and quantification. Mid-turbinate swabs were tested by an in-house polymerase chain reaction (PCR). The panel included, influenza virus $A \& B$, respiratory syncytial virus (RSV), parainfluenza viruses (PIV) 1-3, human metapneumovirus (HMPV), adenovirus, rhinovirus, enterovirus, parechovirus, bocavirus, coronaviruses (COV) OC43, NL63, 229E, HKU1, and mycoplasma. Day 0 samples were initially tested. Where an agent was identified, all samples (day 0 - day 4) were tested in parallel and the cycle threshold value (CT value) converted to $\log _{10}$ to estimate change in viral shedding. CT was capped at 40 as samples with CT values above this level was unlikely to be positive on repeat testing. CT values were converted to $\log _{10}$ using this formula (40-CT of sample)/3.3 (a CT of 3.3 represents a log change in viral load by PCR). Since the baseline sample was tested on two separate occasions, reproducibility of testing was assessed by comparing the two results. Inter-assay variation was expected to be $<0.5 \log _{10}$. Where consecutive samples had undetectable virus, the first sample that was undetectable was treated as the end-point for viral shedding analysis. For the intervention arm, since $\mathrm{NaCl}$ could have an antiviral effect, the day with the lowest shedding when HSNIG was being performed was treated as the end-point. Where HSNIG was stopped earlier than 4 days, since swabs were collected first thing in the morning, the day after HSNIG was stopped was considered the end-point. Hence for the intervention arm, the end-point was day 4 [apart from participant numbers 1, 5, 7, 13, 16, 17, 18 and 19 where it was day $3 ; 2$ (rhinovirus), 14, and 15 where it was day 2; and 11 and 12 where it was day 1]. Two individuals had dual viral infections in their specimen. Both viruses were included for viral shedding analysis.

To determine if there was reduction in viral shedding, the $\log _{10}$ value of the day 0 sample was subtracted from the $\log _{10}$ value of the end-point. Negative values indicate a reduction in viral shedding and positive values indicate an increase in viral shedding. To determine the reduction in viral shedding per day, these values were divided by the number of days of follow-up. The proportion of individuals with reduction in viral shedding by $\geq 0.5 \log _{10} /$ day was then calculated.

Statistical analysis. We used SAS v9.4 software for statistical analysis. For categorical data, we present numbers and percentages. For continuous data, we present mean (standard deviation) or median (interquartile range $(\mathrm{IQR})$ ) as appropriate. Binomial test for the comparisons of proportions was used to examine differences in proportions and presented along with $95 \%$ CI for differences in proportions or chi-square tests depending on the number of groups. To compare differences between treatment arms, two-sample t-tests or Mann-Whitney tests were used, as appropriate.

Participants were considered in the groups to which they were randomised irrespective of treatment received for analysis. Due to the way the data were collected, baseline information was available for all participants. Any subsequent information was the result of participants returning the diary card, potential non-return of diary cards was accounted for by increasing our sample size to allow for drop-outs. In the cases where diary cards were not returned, we do not have any information beyond baseline and therefore we have not used any methods to impute any missing data.

\section{References}

1. van Gageldonk-Lafeber, A. B. et al. A case-control study of acute respiratory tract infection in general practice patients in The Netherlands. Clin Infect Dis 41, 490-497, https://doi.org/10.1086/431982 (2005).

2. Fendrick, A. M., Monto, A. S., Nightengale, B. \& Sarnes, M. The economic burden of non-influenza-related viral respiratory tract infection in the United States. Arch Intern Med 163, 487-494 (2003).

3. Office for National Statistics: Sickness absence in the labour market: 2016. (London, 2017). 
4. Oppong, R. et al. Resource use and costs of treating acute cough/lower respiratory tract infections in 13 European countries: results and challenges. Eur J Health Econ 12, 319-329, https://doi.org/10.1007/s10198-010-0239-1 (2011).

5. Hicks, L. A. et al. Two outbreaks of severe respiratory disease in nursing homes associated with rhinovirus. J Am Geriatr Soc 54, 284-289, https://doi.org/10.1111/j.1532-5415.2005.00529.x (2006).

6. Walter, J. M. \& Wunderink, R. G. Severe Respiratory Viral Infections: New Evidence and Changing Paradigms. Infect Dis Clin North Am 31, 455-474, https://doi.org/10.1016/j.idc.2017.05.004 (2017).

7. Stelzer-Braid, S. et al. Exhalation of respiratory viruses by breathing, coughing, and talking. J Med Virol 81, 1674-1679, https://doi. org/10.1002/jmv.21556 (2009)

8. Muktibodhananda, S. Hatha Yogo Pradipika. Light on Hatha Yoga. Fourth Edition edn, 202-205 (Bihar School of Yoga, India., 2012).

9. Rabone, S. J. \& Saraswati, S. B. Acceptance and effects of nasal lavage in volunteer woodworkers. Occup Med (Lond) 49, 365-369 (1999).

10. Adam, P., Stiffman, M. \& Blake, R. L. Jr. A clinical trial of hypertonic saline nasal spray in subjects with the common cold or rhinosinusitis. Arch Fam Med 7, 39-43 (1998).

11. Slapak, I., Skoupa, J., Strnad, P. \& Hornik, P. Efficacy of isotonic nasal wash (seawater) in the treatment and prevention of rhinitis in children. Arch Otolaryngol Head Neck Surg 134, 67-74, https://doi.org/10.1001/archoto.2007.19 (2008).

12. King, D., Mitchell, B., Williams, C. P. \& Spurling, G. K. Saline nasal irrigation for acute upper respiratory tract infections. Cochrane Database Syst Rev 4, CD006821, https://doi.org/10.1002/14651858.CD006821.pub3 (2015).

13. Koksal, T. et al. Comparison between the use of saline and seawater for nasal obstruction in children under 2 years of age with acute upper respiratory infection. Turk J Med Sci 46, 1004-1013, https://doi.org/10.3906/sag-1507-18 (2016)

14. Speir, R. W. Effect of several inorganic salts on the infectivity of Mengo virus. Proc Soc Exp Biol Med 106, 402-404 (1961).

15. Ramalingam, S. et al. Antiviral innate immune response in non-myeloid cells is augmented by chloride ions via an increase in intracellular hypochlorous acid levels. Sci Rep 8, 13630, https://doi.org/10.1038/s41598-018-31936-y (2018).

16. Jiang, P., Liu, Y., Ma, H. C., Paul, A. V. \& Wimmer, E. Picornavirus morphogenesis. Microbiol Mol Biol Rev 78, 418-437, https://doi. org/10.1128/MMBR.00012-14 (2014).

17. Collins, P. L. \& Graham, B. S. Viral and host factors in human respiratory syncytial virus pathogenesis. J Virol 82, 2040-2055, https:// doi.org/10.1128/JVI.01625-07 (2008).

18. Lessler, J. et al. Incubation periods of acute respiratory viral infections: a systematic review. Lancet Infect Dis 9, 291-300, https://doi. org/10.1016/S1473-3099(09)70069-6 (2009)

19. Munywoki, P. K. et al. Influence of age, severity of infection, and co-infection on the duration of respiratory syncytial virus (RSV) shedding. Epidemiol Infect 143, 804-812, https://doi.org/10.1017/S0950268814001393 (2015).

20. Kelly, M. S. et al. Association of respiratory viruses with outcomes of severe childhood pneumonia in Botswana. PLoS One 10, e0126593, https://doi.org/10.1371/journal.pone.0126593 (2015).

21. Chen, W. J. et al. Epidemiologic, clinical, and virologic characteristics of human rhinovirus infection among otherwise healthy children and adults: rhinovirus among adults and children. J Clin Virol 64, 74-82, https://doi.org/10.1016/j.jcv.2015.01.007 (2015).

22. Mansbach, J. M. et al. Prospective multicenter study of viral etiology and hospital length of stay in children with severe bronchiolitis. Arch Pediatr Adolesc Med 166, 700-706, https://doi.org/10.1001/archpediatrics.2011.1669 (2012).

23. Jartti, T. et al. Hospital length-of-stay is associated with rhinovirus etiology of bronchiolitis. Pediatr Infect Dis J33, 829-834, https:// doi.org/10.1097/INF.0000000000000313 (2014).

24. Marguet, C. et al. In very young infants severity of acute bronchiolitis depends on carried viruses. PLoS One 4, e4596, https://doi. org/10.1371/journal.pone.0004596 (2009).

25. Tano, L. \& Tano, K. A daily nasal spray with saline prevents symptoms of rhinitis. Acta Otolaryngol 124, 1059-1062, https://doi. org/10.1080/00016480410017657 (2004)

26. Jantsch, J. et al. Cutaneous $\mathrm{Na}+$ storage strengthens the antimicrobial barrier function of the skin and boosts macrophage-driven host defense. Cell Metab 21, 493-501, https://doi.org/10.1016/j.cmet.2015.02.003 (2015).

27. Minton, K. Antibacterial immunity: a pinch of salt. Nat Rev Immunol 15, 202, https://doi.org/10.1038/nri3835 (2015).

28. Larios, O. E. et al. Self-collected mid-turbinate swabs for the detection of respiratory viruses in adults with acute respiratory illnesses. PLoS One 6, e21335, https://doi.org/10.1371/journal.pone.0021335 (2011).

29. Templeton, K., Dove, J., Pilarska, A., Serrano, I. \& Ramalingam, S. In 8th European Meeting on Molecular Diagnostics (Netherlands, October 2013).

30. Lu, Y. et al. Viral aetiology in adults with acute upper respiratory tract infection in Jinan, Northern China. Clin Dev Immunol 2013, 869521, https://doi.org/10.1155/2013/869521 (2013).

31. Zhang, G. et al. High incidence of multiple viral infections identified in upper respiratory tract infected children under three years of age in Shanghai, China. PLoS One 7, e44568, https://doi.org/10.1371/journal.pone.0044568 (2012).

32. Eccles, R. et al. Efficacy and safety of iota-carrageenan nasal spray versus placebo in early treatment of the common cold in adults: the ICICC trial. Respir Res 16, 121, https://doi.org/10.1186/s12931-015-0281-8 (2015).

33. Barrett, B. et al. Validation of a short form Wisconsin Upper Respiratory Symptom Survey (WURSS-21). Health Qual Life Outcomes 7, 76, https://doi.org/10.1186/1477-7525-7-76 (2009).

34. Mirsaeidi, M. et al. Video directly observed therapy for treatment of tuberculosis is patient-oriented and cost-effective. Eur Respir J 46, 871-874, https://doi.org/10.1183/09031936.00011015 (2015).

35. Alipanah, N. et al. Adherence interventions and outcomes of tuberculosis treatment: A systematic review and meta-analysis of trials and observational studies. PLoS Med 15, e1002595, https://doi.org/10.1371/journal.pmed.1002595 (2018).

36. Jackson, G. G., Dowling, H. F., Spiesman, I. G. \& Boand, A. V. Transmission of the common cold to volunteers under controlled conditions. I. The common cold as a clinical entity. AMA Arch Intern Med 101, 267-278 (1958).

\section{Acknowledgements}

We would like to acknowledge the Edinburgh Clinical Research Facility where recruitment was done. We would like to thank the trial nurses particularly Jill Steven, Audrey Kuchnowski for helping with the study, Allan Walker and David Buchanan from Edinburgh Clinical Trials Unit for designing and maintain the database, Glen Merrit from the finance department, Philip Tanswell (Cornish Sea Salt Co.) for providing the sea-salt, and Santina Castriciano from Copan, Italy for providing us with mid-turbinate swabs and eNAT transport medium. Our thanks to Midlothian and Edinburgh councils and schools and NHS Lothian for helping us advertise the study and most importantly, the participants for taking part in the study. Sandeep Ramalingam is financially supported by NHS Research Scotland (NRS) and the Scottish Chief Scientist Office (CSO). Aziz Sheikh is supported by the FARR institute, which is funded by a consortium of funders led by the MRC. This work was supported by the Edinburgh and Lothians Health Foundation [Reference 10-303 to S.R.]. 


\section{Author Contributions}

S.R. Principle Investigator. Was involved in designing, conducting, analysing and writing the manuscript. C.G. Involved in designing, analysing and writing the manuscript. J.D. Involved in testing and reviewing the manuscript. L.M. Involved in designing and reviewing the manuscript. A.S. Involved in designing and writing the manuscript.

\section{Additional Information}

Supplementary information accompanies this paper at https://doi.org/10.1038/s41598-018-37703-3.

Competing Interests: The authors declare no competing interests.

Publisher's note: Springer Nature remains neutral with regard to jurisdictional claims in published maps and institutional affiliations.

(c) (i) Open Access This article is licensed under a Creative Commons Attribution 4.0 International License, which permits use, sharing, adaptation, distribution and reproduction in any medium or format, as long as you give appropriate credit to the original author(s) and the source, provide a link to the Creative Commons license, and indicate if changes were made. The images or other third party material in this article are included in the article's Creative Commons license, unless indicated otherwise in a credit line to the material. If material is not included in the article's Creative Commons license and your intended use is not permitted by statutory regulation or exceeds the permitted use, you will need to obtain permission directly from the copyright holder. To view a copy of this license, visit http://creativecommons.org/licenses/by/4.0/.

(C) The Author(s) 2019 